This is the accepted manuscript version of the following article: Advanced Functional Materials, Volume 25, Issue 32 ,

Pages 5178-5183, which has been published in final form at

http://onlinelibrary.wiley.com/doi/10.1002/adfm.201501900/abstract

DOI: 10.1002/adfm.((please insert DOI)

\title{
Vectorially Imprinted Hybrid Nanofilm for Acetylcholinesterase Recognition
}

K. J. Jetzschmann, G. Jágerszki, D. Dechtrirat, A. Yarman, N. Gajovic-Eichelmann, H.-D.Gilsing, B.Schulz, R.E. Gyurcsányi ${ }^{*}$ and F. W. Scheller*

K. J. Jetzschmann M.Sc., Dr. A. Yarman, Prof. Dr. F. W. Scheller

Institute of Biochemistry and Biology

University of Potsdam

Karl-Liebknecht Strasse 24-25, 14476 Potsdam (Germany)

Dr. N. Gajovic-Eichelmann, Prof. Dr. F. W. Scheller

Fraunhofer IZI-BB

Am Mühlenberg 13, 14476 Potsdam (Germany)

Fax: (+49) 331-58187-299

E-mail: fschell@uni-potsdam.de

Dr. D. Dechtrirat

Department of Materials Science, Faculty of Science, Kasetsart University, 50 Phaholyothin Rd., Ladyao,

Chatuchak, Bangkok 10900, Thailand

Dr. H.-D. Gilsing, Dr. B. Schulz

IDM

Kantstrasse 55,14513 Teltow (Germany)

Gyula Jágerszki M.Sc., Prof. Dr. Róbert E. Gyurcsányi

MTA-BME "Lendület" Chemical Nanosensors Research Group

Department of Inorganic and Analytical Chemistry

Budapest University of Technology and Economics

Szt. Gellért tér 4, 1111 Budapest, Hungary

Fax: (+36) 14633408

E-mail: robertgy@mail.bme.hu

Keywords: biomimetic sensors, molecularly imprinted electropolymers, acetylcholinesterase, peripheral anionic site, propidium 


\begin{abstract}
Effective recognition of enzymatically active tetrameric acetylcholinesterase (AChE) was accomplished by a hybrid nanofilm composed of a propidium-terminated self-assembled monolayer (Prop-SAM) which binds AChE via its peripheral anionic site (PAS) and an ultra-thin electrosynthesized molecularly imprinted polymer (MIP) cover layer of a novel carboxylate-modified derivative of 3,4propylenedioxythiophene. The rebinding of the AChE to the MIP/Prop-SAM nanofilm covered electrode was detected by measuring in situ the enzymatic activity. The oxidative current of the released thiocholine was dependent on the $\mathrm{AChE}$ concentration from ca. $0.04 \mu \mathrm{M}$ to $0.4 \mu \mathrm{M}$. An imprinting factor of 9.9 was obtained for the hybrid MIP, which is among the best values reported for protein imprinting. The dissociation constant characterizing the strength of the MIP-AChE binding was $4.2 \times 10^{-7} \mathrm{M}$ indicating the dominant role of the PAS-Prop-SAM interaction, while the benefit of the MIP nanofilm covering the Prop-SAM layer was the effective suppression of the cross-reactivity towards competing proteins as compared with the Prop-SAM. The threefold selectivity gain provided by (i) the "shapespecific" MIP filter, (ii) the propidium-SAM, (iii) signal generation only by the AChE bound to the nanofilm show promise for assessing AChE activity levels in CSF.
\end{abstract}

\title{
1. Introduction
}

Molecularly imprinted polymers (MIPs) have been successfully developed for low molecular weight substances by polymerizing functional monomers in the presence of a target molecule, the template. ${ }^{[1,2]}$ 
Upon removal of the template, cavities complementary to the template in shape, size and functionality are left behind. The application of this simple concept for complex macromolecular templates like proteins however is still a challenge (see for details Supporting Information). ${ }^{[3-6]}$ An essential prerequisite to generate protein MIPs is to ensure the effective exchange of the target molecule between the polymer and solution phase. Most often this converts in having the protein binding sites confined to the surface of the MIPs by using surface imprinting techniques. ${ }^{[7-13]}$ Uniform orientation of the templates during polymerization is a second prerequisite to generate homogeneous binding sites for proteins and reducing the cross-reactivity of the respective MIPs. ${ }^{[6,14]}$ For this purpose the template should be bound via a sitespecific affinity ligand to the surface, where, as template either the whole protein or a fragment of it (epitope-imprinting) could be used. ${ }^{[15-19]}$ Both approaches could be successfully demonstrated by our group for the imprinting of Cytochrome $c$ as well as the lectin concanavalin $A .{ }^{[20,21]}$ In this respect we have worked out electropolymerization strategies for the generation of ultra-thin MIP films with nanometer precision - based on controlling the charge passed for the film formation - to perfectly match the dimensions of the harbored template proteins. Such electrosynthesized MIPs are inherently compatible with a wide range of transducers.

However, in spite of large efforts, the overall analytical performance of MIPs still lags behind that of antibodies and enzymes, which calls for new strategies and monomers. In this respect here we propose the combined use of a hybrid multilayered architecture for selective protein recognition that consists of an ultrathin protein-imprinted polymer film deposited over a layer of specific receptor. This approach may be used to modulate the selective access of template proteins to a receptor and as such to discriminate between different forms and associations of the target proteins. We show the proof of concept by synthesizing a novel hybrid material for the recognition of acetylcholinesterase (AChE) in which electrosynthesis is used to generate an ultrathin imprinted cover layer to regulate the access of proteins to a surface confined propidium underlayer that is known to bind to the peripheral anionic site (PAS) of AChE. ${ }^{[22]}$ The application of a weak inhibitor such as propidium to orientedly bind AChE via its PAS while the enzymatic activity is conserved is a major advantage as compared with the state of the art for 
protein-MIPs. ${ }^{[23]}$ Furthermore, a novel functional monomer is introduced, i.e., a carboxylate-modified derivative of 3,4-propylenedioxythiophene (ProDOT-COOH) (see Supporting Information for the synthesis). At $\mathrm{pH}=8$, optimal for the activity of $\mathrm{AChE}$, the non-specific interactions with AChE are largely suppressed due to charge repulsion between the negatively charged ProDOT-COOH polymer and $\mathrm{AChE}(\mathrm{IEP}=5.35)$.

This hybrid nanofilm is intended as the first step toward a sensor for the indication of different AChEspecies as potential early diagnostic markers of Alzheimer's disease (AD). ${ }^{[24]}$ Progression of AD is accompanied by an alteration in AChE activity and a change in the glycosilation-pattern. ${ }^{[25]}$ Therefore, a sensor-system that can effectively detect $\mathrm{AChE}$, discriminate between its differently glycated isoforms and simultaneously determine the enzymatic activity would be of great interest. The homotetrameric AChE from electric eel (Electrophorus electricus, PDB-code: 1C2O) is used in this study as a model template since it shares a high sequence similarity of $88.5 \%$ with the human AChE, which also exist mostly as homotetramer in the brain (see Supporting Information).

\section{Results and Discussion}

For the preparation of the hybrid recognition-layer first the self-assembled monolayer with terminal propidium units (Prop-SAM) was formed on a gold electrode to which the template AChE is docked via its PAS (Scheme 1). Then ProDOT-COOH monomer is electropolymerized under charge control to form a conformal polymer film around the oriented AChE templates with precisely defined thickness, which is smaller than the template diameter to enable its free exchange, but thick enough to hold the imprint of AChE. ${ }^{[13,26,27]}$ This tailored synthesis of the hybrid recognition layer with nanometer precision is surprisingly easy to control and more robust than many surface imprinting procedures including transfer of ultra-thin membranes, stamping, and template synthesis of micro- and nanostructures. ${ }^{[10,12,16,28,29]}$

The average thickness of the MIP films $(5.0 \pm 0.4 \mathrm{~nm})$ was significantly larger than that of the respective control polymer (NIP) films $(1.7 \pm 0.5 \mathrm{~nm})$ as determined by atomic force microscopy (AFM). The thicknesses could be very precisely determined by removing a rectangular area of the polymer film 
using the AFM tip in nanolithography mode and then rescanning the same surface in tapping mode to determine the step height of the trench (see Figure S1 and S2). Obviously, the AChE molecules are only partially entrapped by the polymer layer because the protein $(13.2 \times 13.2 \times 5.5 \mathrm{~nm})$ is almost twice as large as the average thickness of the MIP. ${ }^{[30]}$

We have further used AFM to confirm the removal of the template from the MIP as well as its rebinding. For this purpose a glycine- $\mathrm{HCl}$ buffer $(\mathrm{pH} 2.2)$ was used as it may - besides disrupting hydrogen bonds - facilitate the removal of the template by supporting the dissociation of the noncovalently connected dimeric subunits. ${ }^{[30]}$ AFM measurements (Figure 1) showed a marked difference in the surface topography of the MIP before and after removal of AChE. Analyzing line profiles of the recorded AFM images (Figure S3) it was found that the removal of the template leaves behind well distinguishable "cavities". Upon template rebinding these cavities disappeared resulting additionally in the apparition of peaks with an average height of $8.1( \pm 2.1) \mathrm{nm}$ (determined from representative areas of three different MIP-modified electrodes). Accounting also for the depth of the "cavities" (ca. $2 \mathrm{~nm}$ ) that seem to be filled up during template rebinding the ca. $10 \mathrm{~nm}$ height objects can be assigned to be AChE molecules or cluster of molecules as the lateral resolution of the technique is not sufficient for further substantiation.

The presence of active AChE in the electropolymer-covered, AChE-loaded SAM was confirmed by monitoring the enzymatic generation of electroactive thiocholine from acetylthiocholine (ATC) at a potential of $0.3 \mathrm{~V}$ (vs. $\mathrm{Ag} / \mathrm{AgCl})$ (Scheme 1). ${ }^{[31]}$ The amperometric signal of the NIP after electropolymerization is very low and, since the electrode has not been in contact with AChE, is due to the well-known spontaneous hydrolysis of ATC. ${ }^{[32]}$ The enzymatic activity of MIP-sensors after AChEloading and electropolymerization is ca. six times higher than for the NIP indicating the presence of active AChE in the film (Figure 2). After incubation of the MIP in glycine- $\mathrm{HCl}$ buffer the oxidation current of thiocholine is decreased to a level close to that of the NIP. The same treatment of AChE in solution resulted in a comparable decrease of enzymatic activity. Therefore to confirm that the loss of activity is indicative of the template removal and it is not solely an inactivation of the surface bound 
enzymes, rebinding experiments with topographic AFM and substrate conversion measurements were performed in parallel. As shown in Figure 2 by incubation of the MIP in AChE containing solutions $\left(50 \mu \mathrm{g} \mathrm{mL}^{-1}\right)$ the enzyme activity was regained, confirming in agreement with the AFM experiments (Figure S3) the rebinding of AChE to the binding sites of the electropolymerized MIPs. The latter statement is further supported by the fact that the NIP-response did not change significantly during template-removal-rebinding cycles, implying a low non-specific binding of AChE to the electropolymerized ProDOT-COOH surface.

The detection of the target rebinding by measuring the electroactive product of the enzymatic reaction is more specific and convenient than to evaluate changes in the surface loading by other electrochemical means (e.g. redox marker permeation) or by mass-sensitive transducers, e.g., surface plasmon resonance or QCM. However, the latter techniques are compatible with the proposed hybrid nanofilm system and can be considered for template proteins that cannot be detected through their inherent catalytic activity.

The recovery of the enzymatic activity by binding of the target to inactivated AChE which remains entrapped in the MIP by so called template-template interaction is not likely to be significant based on the AFM experiments, which show evidence for target removal and based on the thickness of the AChE layer suggest that the binding is constricted to a monolayer. However, lateral clustering around the bound and active AChE molecules cannot be excluded. ${ }^{[33,34,22]}$

In order to quantitate the concentration dependence of the AChE rebinding to the MIP the oxidation current of ATC after incubation in different AChE-concentrations was measured (Figure 3). Starting at the value of spontaneous hydrolysis the current increased with the AChE concentration approaching saturation above $100 \mu \mathrm{g} \mathrm{mL} \mathrm{m}^{-1}$. In contrast, for the NIP-sensors after equal treatment the current hardly exceeded the level of spontaneous ATC hydrolysis. The significantly higher surface activity upon template rebinding of $\mathrm{AChE}$ on the MIP as compared to the NIP at all concentrations confirms the formation of AChE-specific binding sites during imprinting. The imprinting factor (IF), defined as the ratio of the AChE activity of the MIP and NIP film, respectively, was calculated to be 5.3, based on the oxidation currents at $100 \mu \mathrm{g} \mathrm{mL}^{-1}$ AChE concentration. Taking into account the self-hydrolysis of ATC, 
the blank-value-adjusted $I F$ increases to 9.9 , which is among the best values reported for protein imprinting in literature (see Supporting Information). The dissociation constant $K_{D}$ for the MIP surface was determined by fitting the Langmuir model to be $4.2 \times 10^{-7} \mathrm{M}$, which is in the range of the values reported in literature for the interaction in solution $\left(K_{D}=3-7.1 \times 10^{-7} \mathrm{M}\right) .^{[35-38]}$

In order to characterize the contribution of the interaction of the AChE via its PAS we performed binding experiments in presence of two inhibitors with both the Prop-SAM and Prop-SAM/MIP. The reversible ligand propidium (MW: $668.4 \mathrm{Da}$ ) as well as the amyloid- $\beta$-peptide A $\beta 42$ (peptide of 42 amino acids, MW: $4330 \mathrm{Da}$ ), which is known to bind to a hydrophobic area close to the peripheral anionic binding site, were applied as competitors in solution for AChE binding. ${ }^{[37,38]}$

For this reason Prop-SAM and Prop-SAM/MIP films were incubated in mixtures of one of the inhibitors and $\mathrm{AChE}$ and the resulting enzymatic activity of the films was evaluated in a separate solution containing ATC. For the PAS-specific inhibitor propidium and the peptide A $\beta 42$, the Prop-SAM modified electrode and the Prop-SAM/MIP structure showed similar trends of the decrease in enzymatic activity. It approached the value of spontaneous hydrolysis (Figure 4) whilst in solution AChE is only partially inhibited by propidium. ${ }^{[22,39]}$ This result may indicate that binding of propidium-loaded AChE to both the SAM and the SAM/MIP hybrid is suppressed. Thus rebinding of non-inhibited AChE should be dominated by the interaction between the PAS and the propidium units but not by binding to eventually entrapped target. The close similarity of the $K_{D^{-}}$values for the binding of $\mathrm{AChE}$ to propidium and to the SAM/MIP also supports this assumption. The low contribution of the interaction with the electropolymer may be based on the electrostatic repulsion between the negatively charged AChE and the negatively charged poly-ProDOT-COOH.

The selectivity of the AChE-imprinted hybrid film was investigated in competitive binding experiments using two functionally unrelated proteins with widely different molecular weights but isoelectric points similar to AChE as competitors: bovine serum albumin (BSA, MW: $66.5 \mathrm{kDa}$, IEP 5.2) and urease (MW: 460 kDa, IEP 4.7). MIP-, NIP- and Prop-SAM-modified electrodes were incubated in a solution containing $\mathrm{AChE}$ at a constant concentration of $50 \mu \mathrm{g} \mathrm{mL}^{-1}$ and different concentrations of the 
competing proteins for $20 \mathrm{~min}$. On the NIP covered electrodes the current signal from the thiocholine oxidation was dominated by the rate of the spontaneous hydrolysis of ATC and was practically independent from the applied competitor concentration. On Prop-SAM modified electrodes without an electropolymer film, the current decreased with the concentration of both competitor proteins, approaching the level found with NIP-modified surfaces (Figure 5), competitively suppressing the binding of AChE. On the MIP-modified surface, however, such displacement occurred only to a minor degree and the AChE surface activity stabilized at 75 and $80 \%$ of the competitor-free level in presence of urease and BSA, respectively. These experiments demonstrate clearly the increased selectivity of the Prop-SAM/MIP architecture as compared with the Prop-SAM modified surfaces. This finding shows that the MIP structure on top of the SAM makes the interaction more selective.

\section{Conclusions}

The congruent effects of the PAS binding inhibitors propidium and $A \beta 42$ on the binding of AChE to Prop-SAM and the Prop-SAM/MIP structure indicate that the interaction between AChE and the PropSAM/MIP hybrid is dominated by the interaction of the PAS with the propidium moiety. This interpretation is in line with the coincidence of the affinity constants for the binding of AChE to the PropSAM/MIP surface and to propidium in solution. Thus, very importantly the AChE imprinted polymer layer permits full access to the surface confined propidium layer. The low contribution of the interaction with the MIP can be explained by the electrostatic repulsion between AChE and ProDOT-COOH which are both negatively charged. However, on the other hand the imprinted polymer layer suppresses effectively the binding of negatively charged proteins BSA and urease to the Prop-SAM/MIP while at Prop-SAM layers without a MIP film these proteins show strong competitive inhibition of the AChE binding. Thus the MIP layer has a pronounced selective shielding effect towards foreign proteins as AChE imprints induce preferential binding of $\mathrm{AChE}$ even in relation to the smaller BSA competitor. These results clearly demonstrate the synergism of the hybrid Prop-SAM/MIP films providing the prerequisites to enable their application in complex protein containing samples. In conclusion, the new 
hierarchical hybrid architecture provides a threefold selectivity gain by (i) the propidium-SAM, (ii) "shape-specific" MIP and (iii) signal generation only by the AChE bound to the nanofilm. Thus the approach proposed is likely to be feasible for assessing AChE activity levels in CSF.

\section{Experimental Section}

Before preparation of the MIP, clean gold wires were incubated in lipoic acid (1 mM, in $20 \%$ ethanol) at room temperature for a minimum of $16 \mathrm{~h}$. After removing the excess reagent with ethanol the DCC activated surface was reacted with propidium $(0.34 \mathrm{mM}$ propidium, $0.25 \mathrm{mM}$ DCC in $30 \%$ ethanol) for at least $22 \mathrm{~h}$. AChE-imprinted gold wires were prepared using ProDOT-COOH ( $1 \mathrm{mM}$ in $\left.0.1 \mathrm{M} \mathrm{LiClO}_{4}\right)$ after incubation in AChE $(50 \mu \mathrm{g} / \mathrm{mL}$, from Electrophorus electricus, MW: $280 \mathrm{kDa})$, purchased from Sigma-Aldrich). A potential of $0.75 \mathrm{~V}$ was applied for $5 \mathrm{~s}$ (followed by $5 \mathrm{~s}$ at $0 \mathrm{~V}$ ). Non-imprinted polymer sensors were prepared in an identical manner but in the absence of the template. Template molecules could be removed from the MIP using glycine- $\mathrm{HCl}(50 \mathrm{mM}, \mathrm{pH} 2.2)$ for $16 \mathrm{~min}$. Rebinding of AChE was conducted in sodium phosphate buffer (50 mM, pH 8.0).

The activity of immobilized AChE was determined via enzymatic hydrolysis of ATC $(0.02 \mathrm{mM})$ in the buffer used for rebinding. The product thiocholine was oxidized at the Prop-SAM/MIP covered gold working electrode at a potential of $+0.3 \mathrm{~V}$ vs. $\mathrm{Ag} / \mathrm{AgCl}$.

High resolution AFM measurements of the Prop-SAM/MIP surfaces before and after AChE binding as well as of NIPs was made by Nanosurf FlexAFM using an SSS-NCLR tip (SuperSharpSilicon with a force constant of $48 \mathrm{~N} / \mathrm{m}$, length of $225 \mu \mathrm{m}$, and tip diameter of $\sim 2 \mathrm{~nm}$ ). The $0.5 \times 0.5 \mu \mathrm{m}$ images were made in tapping mode with $40 \mathrm{mV}$ amplitude and 93-97\% set point. The thickness of the polymer films was determined by removing the polymer layer. Curve fitting was performed as detailed in the Supporting information.

\section{Supporting Information}

Supporting Information is available from the Wiley Online Library or from the author. 


\section{Acknowledgement}

The authors would like to thank BMBF (0311993) of Germany for the financial support as well as ERAChemistry $(2014,61133)$. This work is a part of UniCat, the Cluster of Excellence in the field of catalysis coordinated by the Technical University of Berlin and financially supported by Deutsche Forschungsgemeinschaft (DFG) within the framework of the German Excellence Initiative (EXC 314). The Hungarian party acknowledges the support of the Lendület program of the Hungarian Academy of Sciences.

Received: ((will be filled in by the editorial staff))

Revised: ((will be filled in by the editorial staff)) Published online: ((will be filled in by the editorial staff))

[1] K. Haupt, K. Mosbach, Trends Biotechnol.1998, 16, 468.

[2] O. Hayden, P. A. Lieberzeit, D. Blaas, F.L. Dickert, Adv. Funct. Mater. 2006, 16, 1269.

[3] A. Bossi, F. Bonini, A. P. F. Turner, S. A. Piletsky, Biosens. Bioelectron. 2007, 22, 1131.

[4] T. Takeuchi, T. Hishiya, Org. Biomol. Chem.2008, 6, 2459.

[5] Y. Ge, A. P. F. Turner, Trends Biotechnol.2008, 26, 218.

[6] M. J. Whitcombe, I. Chianella, L. Larcombe, S. A. Piletsky, J. Noble, R. Porter, A. Horgan, Chem. Soc. Rev.2011, 40, 1547.

[7] A. Menaker, V. Syritski, J. Reut, A. Öpik, V. Horváth, R. E. Gyurcsányi, Adv. Mater. 2009, 21, 2271.

[8] G. Lautner, J. Kaev, J. Reut, A. Öpik, J. Rappich, V. Syritski, R. E. Gyurcsányi, Adv. Funct. Mater.2011, 21, 591.

[9] A. Nematollahzadeh, W. Sun, C. S. A. Aureliano, D. Lütkemeyer, J. Stute, M. J. Abdekhodaie, A. Shojaei, B. Sellergren, Angew. Chem. 2011, 123, 515-518; Angew. Chem. Int. Ed. 2011, 50, 495.

[10] J. Bognár, J. Szűcs, Z. Dorkó, V. Horváth, R. E. Gyurcsányi, Adv. Funct. Mater. 2013, 23, 4703.

[11] P. Çakir, A. Cutivet, M. Resmini, B. Tse Sum Bui, K. Haupt, Adv. Mater. 2013, 25, 1048.

[12] F. L. Dickert, O. Hayden, R. Bindeus, K. J. Mann, D. Blaas, E. Waigmann, Anal. Bioanal. Chem.2004, 378, 1929.

[13] A. Yarman, F. W. Scheller, Angew. Chem. Int. Edit.2013, 52, 11521.

[14] Y. Kamon, R. Matsuura, Y. Kitayama, T. Ooya, T. Takeuchi. Polymer Chem. 2014, 5,4764. 
[15] A. Rachkov, N. Minoura, J. Chromatogr. A2000, 889, 111.

[16] H. Nishino, C. S. Huang, K. J. Shea, Angew. Chem.2006, 118, 2452-2456; Angew. Chem. Int. Ed. 2006, 45, 2392.

[17] D. F. Tai, M. H. Jhang, G. Y. Chen, S. C. Wang, K. H. Lu, Y. D. Lee, H. T. Liu, Anal. Chem. 2010, 82, 2290.

[18] G. Ertürk, L. Uzun, M. A. Tümer, R. Say, A. Denizli, Biosens. Bioelectron. 2011, $28,97$.

[19] C. H. Lu, Y. Zhang, S. F. Tang, Z. B. Fang, H. H. Yang, X. Chen, G. N. Chen, Biosens. Bioelectron. 2012, 31, 439.

[20] D. Dechtrirat, K. J. Jetzschmann, W.F. M. Stöcklein, F.W. Scheller, N. Gajovic-Eichelmann, Adv. Funct. Mater.2012, 22, 5231.

[21] D. Dechtrirat, N. Gajovic-Eichelmann, F. F. Bier, F.W. Scheller, Adv. Funct. Mater.2013, 24, 2233.

[22] C. Teller, J. Halámek, A. Makower, D. Fournier, H. Schulze, F. W. Scheller, Sens. Actuators, B. 2006, 113, 214.

[23] C. Zheng, X. L. Zhang, W. Liu, B. Liu, H. H. Yang, Z. A. Lin, G. N. Chen. Adv. Mat. 2013, 25, 5922.

[24] F. J. Carvajal, N. C. Inestrosa, Front. Mol. Neurosci.2011, 14, 1.

[25] J. Sáez-Valero, M. S. Barquero, A. Marcos, C. A. McLean, D. H. Small, J. Neurol. Neurosurg. Psychiatry,2000, 69, 664.

[26] C. Malitesta, E. Mazzotta, R. A. Picca, A. Poma, I. Chianella, S. A. Piletsky, Anal. Bioanal. Chem. 2012, 402, 1827.

[27] P. S. Sharma, A. Pietrzyk-Le, F. D'Souza, W. Kutner, Anal. Bioanal. Chem. 2012, 402, 3177.

[28] G. Sener, E. Ozgur, A. Y. Rad, L. Uzun, R. Sayd A. Denizli, Analyst. 2013,138, 6422.

[29] D. Cai, L. Ren, H. Zhao, C. Xu, L. Zhang, Y. Yu, H. Wang, Y. Lan, M. F. Roberts, J. H. Chuang, M. J. Naughton, Z. Ren, T. C. Chiles, Nat. Nano. 2010, 5, 597.

[30] Y. Bourne, J. Grassi, P. E. Bougis, P. Marchot, J. Biol. Chem. 1999, 274, 30370.

[31] J. Halámek, C. Teller, A. Makower, D. Fournier, F. W. Scheller, Electrochim. Acta. 2006, 51, 5174.

[32] D. B. Goldstein, J. Bacteriol.1959, 78, 695.

[33] N. Lavignac, K. R Brain, C. J. Allender, Biosens. Bioelectron. 2006, 22, 138.

[34] R. M. Garcinuno, I. Chianella, A. Guerreiro, I. Mijangos, E. V. Piletska, M. J. Whitcombe, S. A. Piletsky, Soft Matter. 2009, 5, 311.

[35] N. Nunes-Tavares, A. Nery da Matta, C.M. Batista e Silva, G.M.N. Araújo, S.R.W. Louro, A. Hassón-Voloch, Int. J. Biochem. Cell Biol. 2002, 34, 1071.

[36] P. Taylor, S. Lappi, Biochemistry. 1975, 14, 1989. 
[37] G. V. De Ferrari, M. A. Canales, I. Shin, L. M. Weiner, I. Silman, N. C. Inestrosa, Biochemistry.2001, 40, 10447.

[38] O. Di Pietro, F. J. Pérez-Areales, J. Juárez-Jiménez, A. Espargaró, M. V. Clos, B. Pérez, R. Lavilla, R. Sabaté, F. J. Luque, D. Muñoz Torrero, Eur. J. Med. Chem.2014; 84, 107.

[39] W. D. Mallender, T. Szegletes, T. L. Rosenberry, J. Biol. Chem. 1999, 274, 8491. 


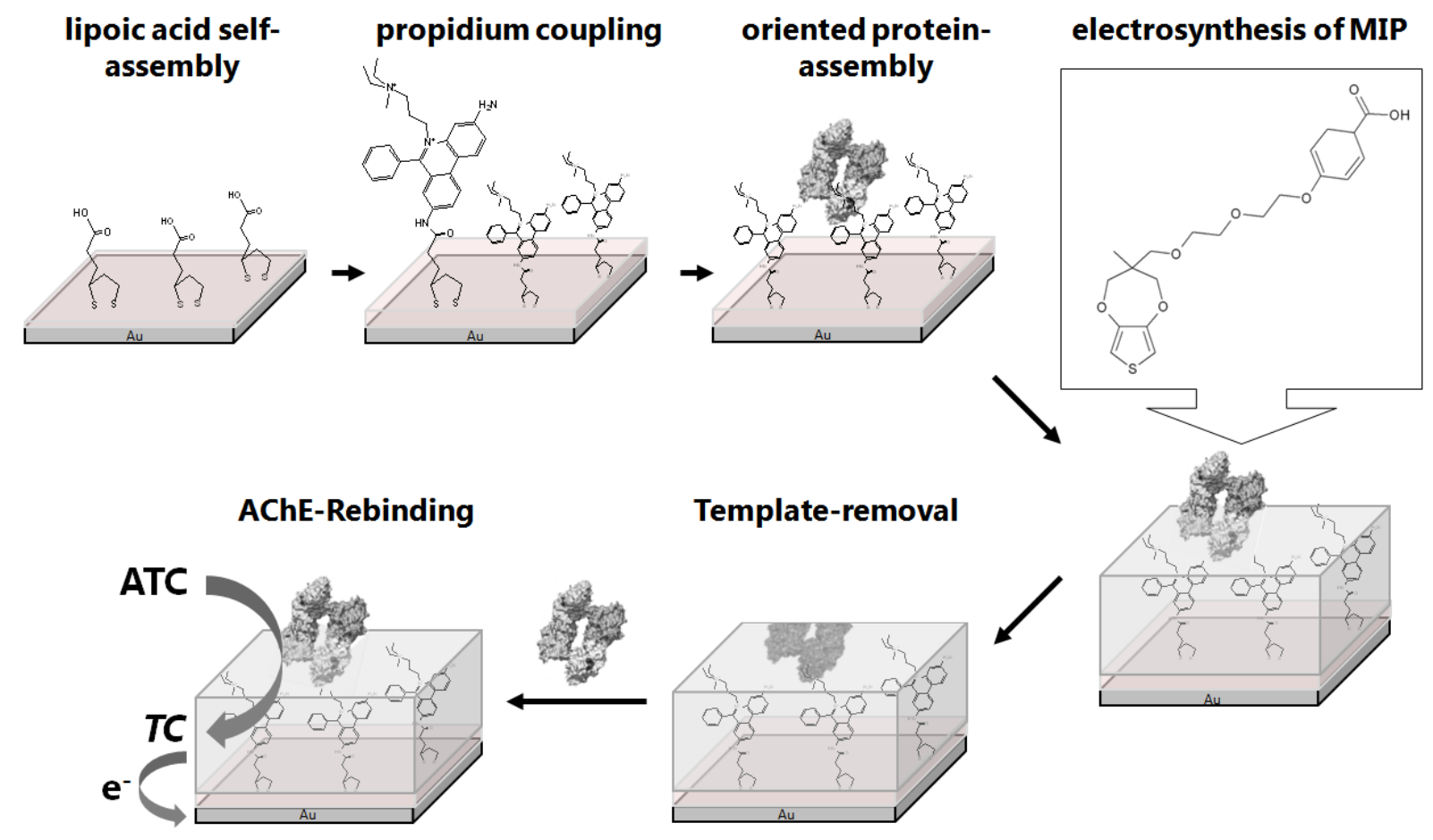

Scheme 1. Schematic representation of the preparation of the vectorially imprinted polymer for AChE by using a propidium self-assembled monolayer on gold in hybrid architecture with an electrosynthesized polymer film from ProDOT-COOH monomer (inset). For the synthesis of the propidium-self assembled monolayer a lipoic-acid-SAM was first formed on a gold electrode to which the propidium was coupled using N,N'-dicyclohexylcarbodiimide (DCC) activation. The AChE is reversibly attached to the PropSAM through its PAS region and then a ca. $5 \mathrm{~nm}$ thin polymer is deposited, which after removal of the AChE template generates binding sites. The rebinding of the template is detected via the generation of thiocholine (TC) from acetylthiocholine (ATC) which is oxidized at the underlying gold electrode. Nonimprinted polymer controls were prepared in the same manner but without loading the Prop-SAM with AChE before the electropolymerization of ProDOT-COOH. 


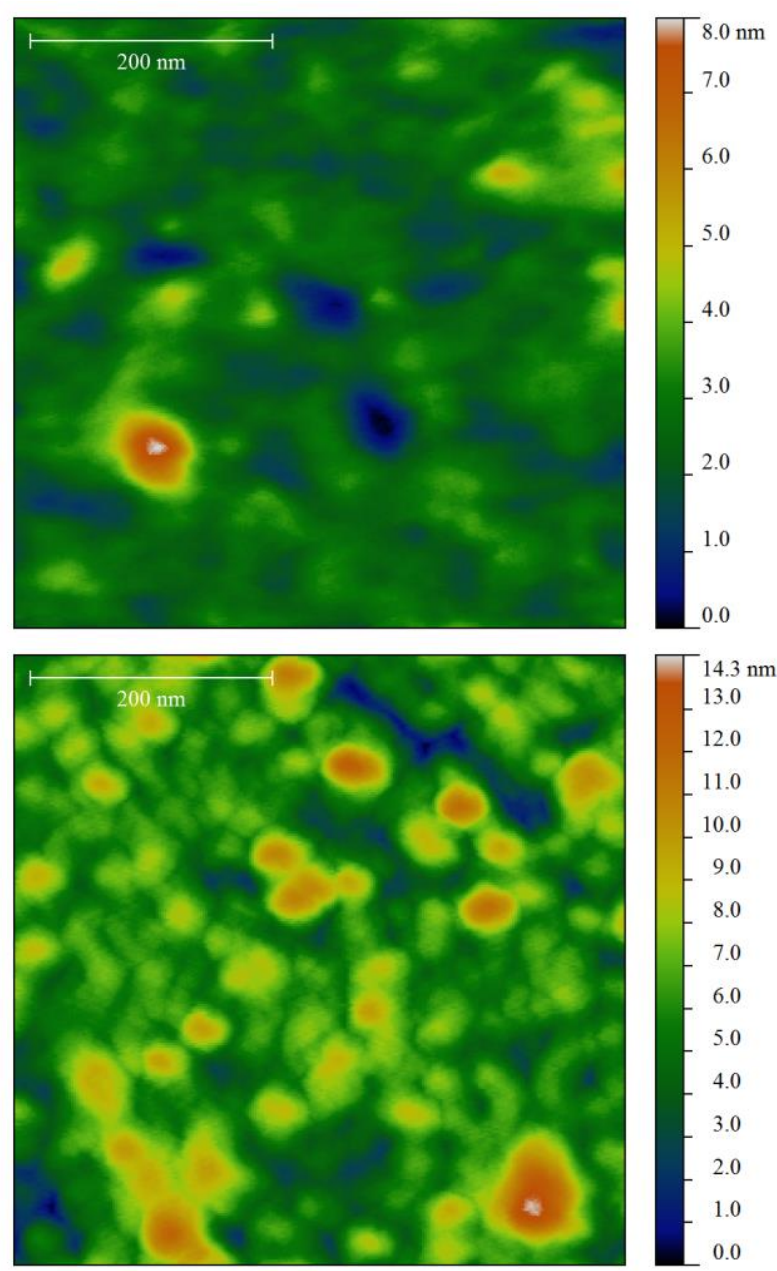

Figure 1. AFM-topography of MIP surfaces with no AChE bound (up) and after incubation with AChE (bottom). 


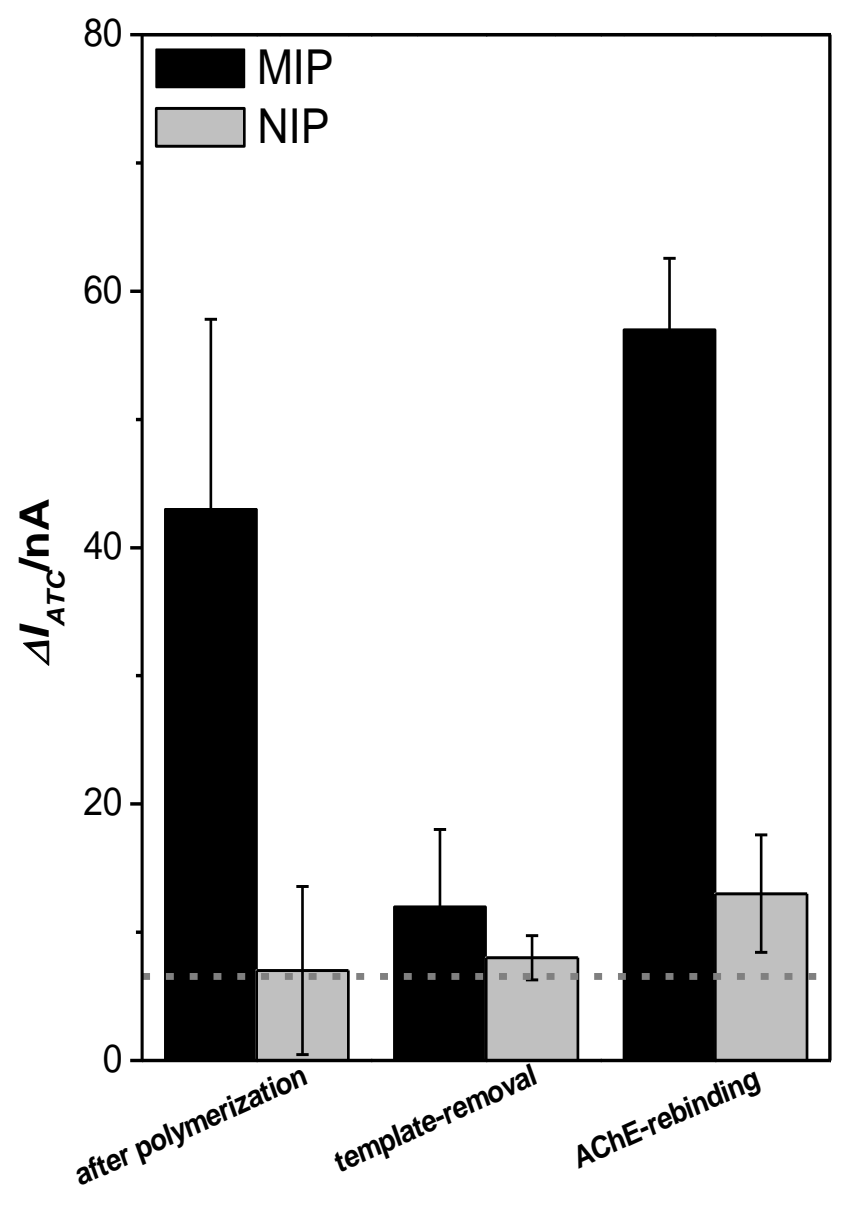

Figure 2. Comparison of the AChE activity of MIP and NIP films after electropolymerization, template removal and AChE-rebinding $\left(50 \mu \mathrm{g} \mathrm{mL}^{-1}\right)$. The AChE activity was determined in a $0.02 \mathrm{mM}$ ATCsolution by measuring the oxidative current of thiocholine at $0.3 \mathrm{~V}$. The dashed line indicates the level of spontaneous self-hydrolysis of ATC. 


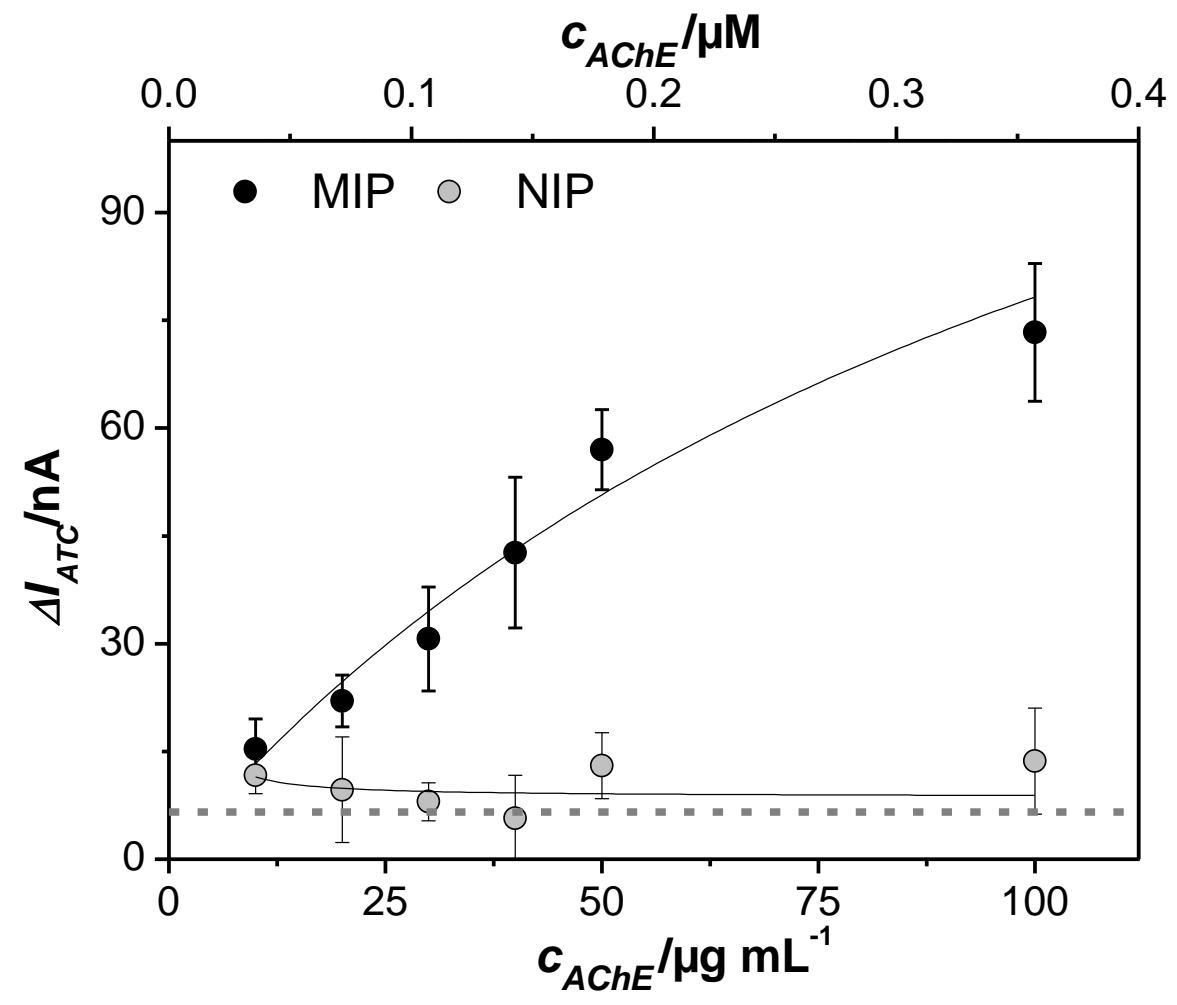

Figure 3. Amperometric response of MIP and NIP covered electrodes as a function of the AChEconcentration after rebinding. The current values reflect the catalytic activity of the bound AChE. The dashed line indicates the level of spontaneous self-hydrolysis of ATC. 

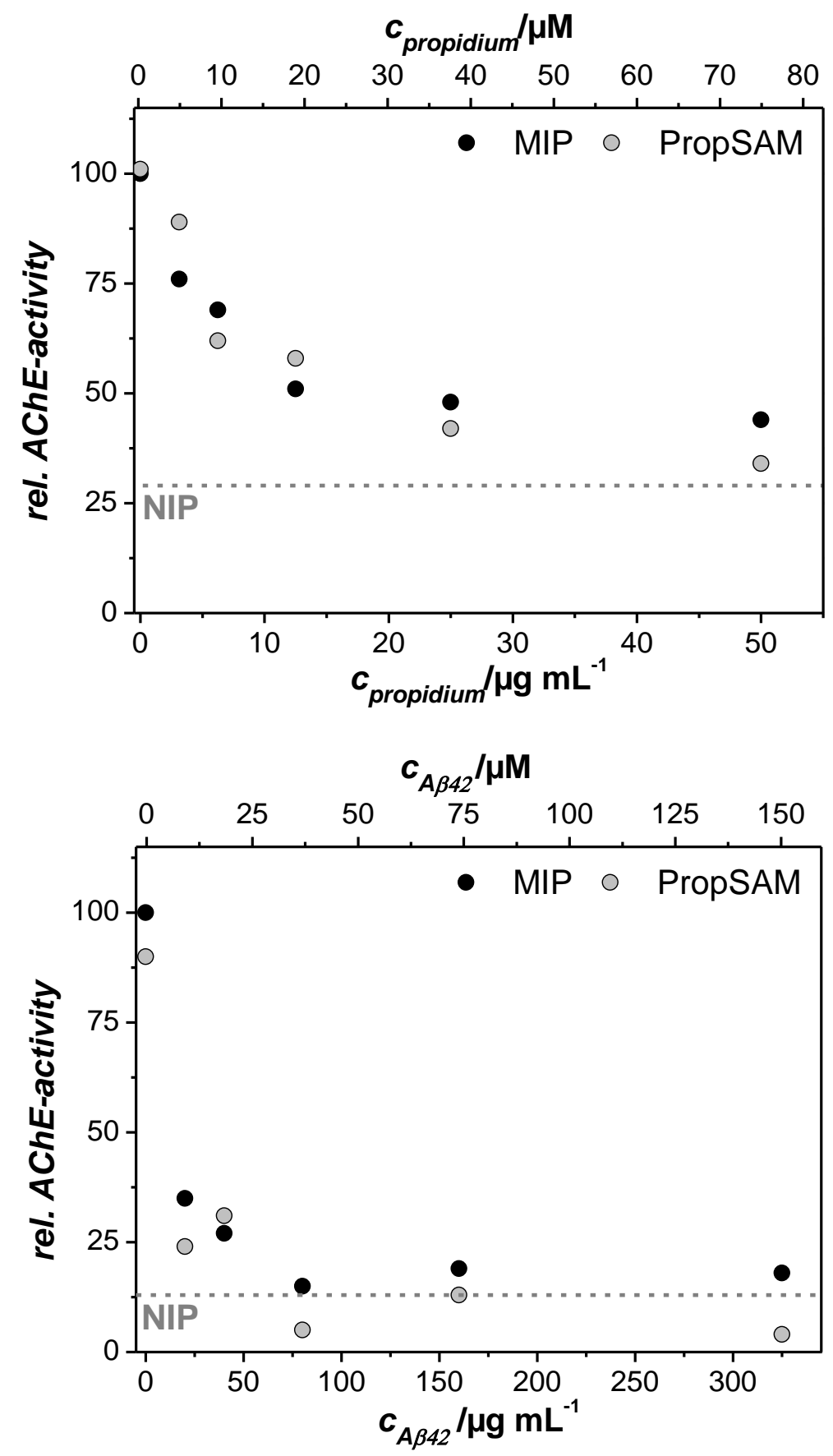

Figure 4. Competitive decrease of AChE binding to Prop-SAM and Prop-SAM/MIP surfaces by the specific inhibitors propidium (top) and A $\beta 42$ peptide (bottom) (both inhibitors bind to the PAS site of AChE). The dashed lines indicate the level of NIP response. 

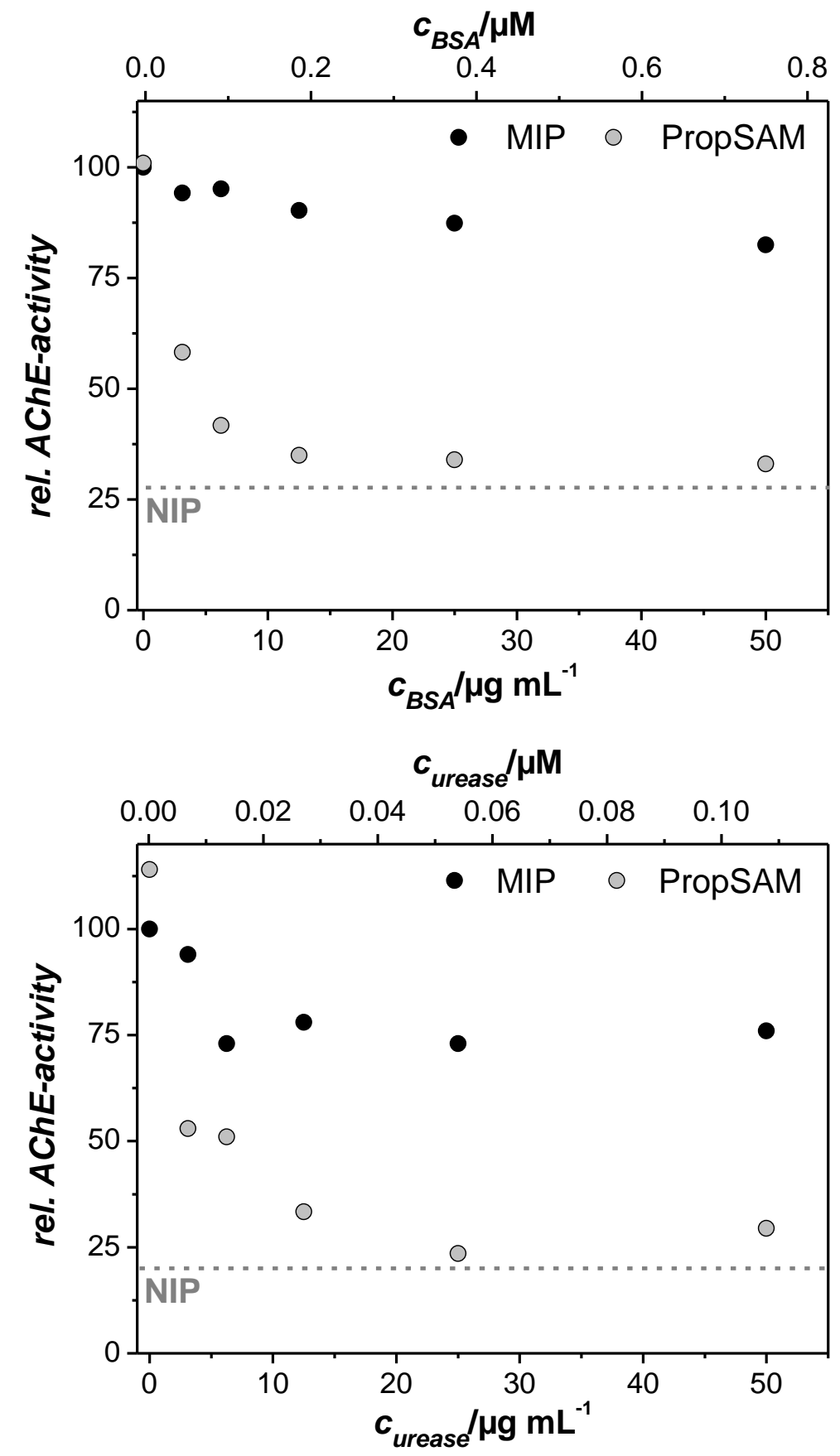

Figure 5. Competitive binding of negatively charged proteins to Prop-SAM and Prop-SAM/MIP surfaces. BSA (top, MW: $66.5 \mathrm{kDa}$, IEP 5.2) and urease (bottom, MW: $460 \mathrm{kDa}$, IEP 4.7) were varied in concentration, while AChE was added at $50 \mu \mathrm{g} \mathrm{mL}^{-1}$. The dashed lines indicate the level of NIP response. 